\title{
Synthesis and In-vitro Activity of Some New Class of Thiazolidinone and Their Arylidene Derivatives
}

\author{
Nareshvarma Seelam* and S. P. Shrivastava \\ Heterocyclic Research Laboratory, Department of Chemistry, Dr. H. S. Gour University, Sagar, M.P, India-470003 \\ *E-mail: utdnaresh@yahoo.co.in \\ Received July 26, 2011, Accepted September 15, 2011
}

\begin{abstract}
In an attempt to find a new class of anti microbial agents, a series of thiazolidinone and their 5-arylidene derivatives containing 4-(4-methyl benzamido)-benzoyl moiety were synthesized via the reaction of benzocaine with appropriate chemical reagents. These compounds were screened for their antibacterial activity against Gram-positive bacteria (Bacillus subtilis and Bacillus thuringiensis), Gram-negative bacteria (Escherichia coli, Pseudomonas aeruginosa) and antifungal activity against Botrytis fabae, Fusarium oxysporan and Candida albicans. On the other hand the synthesized compounds were also screened for their anti tubercular activity. IR, ${ }^{1} \mathrm{H}$ NMR, ${ }^{13} \mathrm{C}$ NMR and MS spectral analyses established the structures of the newly synthesized compounds. The results revealed that some of these compounds have shown promising antimicrobial and anti tubercular activity in comparison with standard drugs.
\end{abstract}

Key Words : Benzocaine, $p$-Methylbenzoyl chloride, Thiazolidinone, Antimicrobial activity, Anti tuberculosis activity

\section{Introduction}

One of the major objectives of organic and medicinal chemistry is the design, synthesis and production of molecules, which are having highly therapeutic nature. The rapidly increasing resistance of pathogenic bacteria towards available antibiotics is a worldwide problem, as well as fungal infections continue to increase rapidly in the number of immuno compromised patients. So, the design of new molecules to deal with resistant bacteria and fungi has become one of the most important areas of antimicrobial research today.

Since the discovery of heterocyclic nucleus the chemistry of thiazolidinones and their allied compounds continue to draw attention of organic chemists due to their various biological activities such as antibacterial, ${ }^{1-4}$ anticancer, ${ }^{5}$ antitubercular, ${ }^{6,7}$ antifungal, ${ }^{8}$ anti-inflammatory, ${ }^{9}$ antiviral, ${ }^{10}$ analgesic. ${ }^{11}$ Thiazolidinone and their arylidene derivatives are also having highly therapeutic nature such as antioxidants, ${ }^{12}$ antitumor agents, ${ }^{13}$ agricultural fungicides. ${ }^{14}$

In view of these above findings it was thought of interest to synthesize some new thiazolidinone and their chalcone derivatives having benzoyl moiety by conventional methods. In this present work we report the synthesis and anti microbial studies of (Z)- $N$-(5-(substitutedbenzylidene)-4oxo-2-phenylthiazolidin-3-yl)-4-(4-methylbenzamido)benzamide derivatives.

\section{Experimental}

All melting points were measured on open capillary method. IR spectra were recorded for $\mathrm{KBr}$ disc on Schimadzu-8400 FT IR spectrophotometer. ${ }^{1} \mathrm{H}$ NMR and
${ }^{13} \mathrm{C}$ NMR spectra were measured on a Bruker Avance II 400 spectrometer, operating at 400, $100.6 \mathrm{MHz}$ respectively. Chemical shifts $(\delta)$ are reported in ppm and TMS as an internal standard. Reactions were monitored by thin layer chromatography (TLC) on silica gel, plates were visualizing with ultraviolet light (or) iodine. Column chromatography was performed on Merck silica gel 60 (0.043-0.060 mm).

General Procedure for the Synthesis of 4-(4-methylbenzoylamino)-benzoic Acid Ethyl Ester (1). To a solution of benzocaine $(0.01 \mathrm{~mol})$ in dry ether $(50 \mathrm{~mL}), p$-methyl benzoyl chloride $(0.01 \mathrm{~mol})$ was added drop by drop at $0{ }^{\circ} \mathrm{C}$. The reaction mixture was stirred for $4 \mathrm{~h}$, the resulting solid was filtered, dried and recrystallised to obtain compound (1).

IR $\left(\mathrm{KBr}, v_{\max }, \mathrm{cm}^{-1}\right) 3340.68(\mathrm{~N}-\mathrm{H}), 3055.35(\mathrm{C}-\mathrm{H}$ in hetero aromatic ring), $3042.89(\mathrm{Ar}-\mathrm{H}), 2943.18(\mathrm{C}-\mathrm{H}$ in $\left.\mathrm{CH}_{3}\right), 2907.16\left(\mathrm{C}-\mathrm{H}\right.$ in $\left.\mathrm{CH}_{2}\right), 1639.71(\mathrm{C}=\mathrm{O}$ in ester $)$, $1307.73(\mathrm{C}-\mathrm{N}) ;{ }^{1} \mathrm{H} \mathrm{NMR}\left(400 \mathrm{MHz}, \mathrm{CDCl}_{3}\right) \delta$ 7.38-7.72 (m, $8 \mathrm{H}, \mathrm{Ar}-\mathrm{H}), 8.12(\mathrm{~s}, 1 \mathrm{H},-\mathrm{CONH}), 1.40(\mathrm{t}, 3 \mathrm{H}, J=7.0 \mathrm{~Hz}$, $\left.\mathrm{CH}_{3}\right), 3.85$ (q, 2H, $\left.J=6.5 \mathrm{~Hz}, \mathrm{CH}_{2}\right) ;{ }^{13} \mathrm{C} \mathrm{NMR}(100 \mathrm{MHz}$, $\left.\mathrm{CDCl}_{3}\right) \delta 163.7(\mathrm{C}=\mathrm{O}), 165.1(\mathrm{C}=\mathrm{O}), 126.5,129.1,129.8$, 131.9, 142.2 (aromatics), $25.3\left(\mathrm{CH}_{3}\right)$.

General Procedure for the Synthesis of $N$-(4-hydrazinocarbonyl-phenyl)-4-methyl-benzamide (2). Compound 1 $(0.01 \mathrm{~mol})$ and hydrazine hydrate $(0.02 \mathrm{~mol})$ in ethanol $(20$ $\mathrm{mL}$ ) was refluxed for about $5 \mathrm{~h}$ on a steam bath. After cooling the resulting solid was filtered, dried and recrystallized to obtain compound 2. Pinkish white solid, yield: $75 \%$, mp 195-198 ${ }^{\circ} \mathrm{C}$; IR (KBr, $\left.v_{\max }, \mathrm{cm}^{-1}\right), 3311,3369\left(-\mathrm{NHNH}_{2}\right)$, 3042.81 (Ar-H), $2942.16\left(\mathrm{CH}_{3}\right), 1661.73(\mathrm{C}=\mathrm{O}$ of amide), ${ }^{1} \mathrm{H}$ NMR $\left(400 \mathrm{MHz}, \mathrm{CDCl}_{3}\right) \delta 3.90\left(\mathrm{~s}, 2 \mathrm{H},-\mathrm{NH}_{2}\right), 7.35-7.81$ (m, 8H, Ar-H), 7.91 (s, 1H, -CONH); ${ }^{13} \mathrm{C}$ NMR (100 MHz, $\left.\mathrm{CDCl}_{3}\right) \delta 26.4\left(\mathrm{CH}_{3}\right), 165.2,169.1(\mathrm{C}=\mathrm{O}), 119.8,126.8$, 131.1, 139.8 (aromatics). 
General Procedure for the Synthesis of $N$-[4-(benzylidenehydrazinocarbonyl)phenyl]-4-methyl-benzamide (3). A mixture of compound $2(0.01 \mathrm{~mol})$, benzaldehyde $(0.01 \mathrm{~mol})$ and 2,3-drops of glacial acetic acid in ethanol (20 $\mathrm{mL}$ ) was refluxed on a water bath for about $6 \mathrm{~h}$. The solvent was removed and the residue recrystallized from chloroformmethanol mixture to yield the required compound (3). Brown solid, yield: $72 \%$, mp $210-212{ }^{\circ} \mathrm{C}$, IR $\left(\mathrm{KBr}, v_{\max }\right.$, $\left.\mathrm{cm}^{-1}\right)$; $3341.72(\mathrm{~N}-\mathrm{H}), 3042.16(\mathrm{Ar}-\mathrm{H}), 2942.16(\mathrm{CH}$ of $\left.\mathrm{CH}_{3}\right), 1651.8(\mathrm{C}=\mathrm{O}$ of amide $), 1526.19(\mathrm{C}=\mathrm{N}), 1307.73(\mathrm{C}-$ $\mathrm{N})$; ${ }^{1} \mathrm{H}$ NMR (400 MHz, $\left.\mathrm{CDCl}_{3}\right) \delta$ 7.01-7.8 (m, 13H, Ar-H), $8.03(\mathrm{~s}, 1 \mathrm{H}, \mathrm{CONH}), 5.91(\mathrm{~s}, 1 \mathrm{H},=\mathrm{CH}), 3.25\left(\mathrm{~s}, 3 \mathrm{H}, \mathrm{CH}_{3}\right)$; ${ }^{13} \mathrm{C}$ NMR $\left(100 \mathrm{MHz}, \mathrm{CDCl}_{3}\right) \delta 165.6(\mathrm{C}=\mathrm{O}), 64.8(=\mathrm{CH})$, $26.4\left(\mathrm{CH}_{3}\right), 119.8,127.5,128.8,130.2,140.5$ (aromatics).

4-Methyl-N-(4-((4-oxo-2-phenylthiazolidin-3-yl)carbamoyl)phenyl)benzamide (4). To a solution of compound $\mathbf{3}$ $(0.005 \mathrm{~mol})$ in DMF $(15 \mathrm{~mL})$ was added mercapto acetic acid $(0.005 \mathrm{~mol})$ and $\mathrm{ZnCl}_{2}(0.5 \mathrm{~g})$ and the reaction mixture was refluxed for $8 \mathrm{~h}$, cooled and poured in to crushed ice, the separate solid was filtered and washed with $10 \% \mathrm{NaHCO}_{3}$. The crude product was dried and recrystallized from DMF to obtain the desired compound (4). Pale yellow crystals, yield: 75.66\%; mp 230-232 ${ }^{\circ} \mathrm{C}$; IR (KBr, $\left.v_{\max }, \mathrm{cm}^{-1}\right)$; $3341.98(\mathrm{~N}-$ $\mathrm{H}), 3046.19(\mathrm{Ar}-\mathrm{H}), 2948.06\left(\mathrm{CH}_{3}\right), 2914.93\left(\mathrm{C}-\mathrm{H}\right.$ in $\left.\mathrm{CH}_{2}\right)$, 1640.0 (ring $\mathrm{C}=\mathrm{O}), 1628.71(\mathrm{C}=\mathrm{O}$ of amide), $681.72(\mathrm{C}-\mathrm{S})$; ${ }^{1} \mathrm{H} \mathrm{NMR}\left(400 \mathrm{MHz}, \mathrm{CDCl}_{3}\right) \delta 3.60\left(\mathrm{~s}, 2 \mathrm{H},-\mathrm{CH}_{2}\right.$ in ring), 3.70 (d, 1H, $J=15.8 \mathrm{~Hz}, \mathrm{CH}-\mathrm{Ar}), 7.01-7.71$ (m, 13H, Ar-H), $8.08(\mathrm{~s}, 1 \mathrm{H},-\mathrm{CONH}), 3.25\left(\mathrm{~s}, 3 \mathrm{H}, \mathrm{CH}_{3}\right) ;{ }^{13} \mathrm{C} \mathrm{NMR}(100$ $\left.\mathrm{MHz}, \mathrm{CDCl}_{3}\right) \delta 31.63$ (ring $\left.\mathrm{S}-\mathrm{CH}_{2}\right), 54.98(-\mathrm{CH}), 119.8$, $128.4,129.9,130.5,141.8$ (aromatics), 163.18 (amide$\mathrm{C}=\mathrm{O}), 172.35$ (ring- $\mathrm{C}=\mathrm{O}$ ).

(Z)- $N$-(5-(Substitutedbenzylidene)-4-oxo-2-phenylthiazolidin-3-yl)-4-(4-methylbenzamido) benzamide (5). A mixture of compound $4(0.005 \mathrm{~mol})$, respective aldehyde $(0.005 \mathrm{~mol})$ and anhydrous $\mathrm{CH}_{3} \mathrm{COONa}(0.005 \mathrm{~mol})$ in anhydrous glacial acetic acid $(50 \mathrm{~mL})$ was refluxed for $3 \mathrm{~h}$. The reaction mixture was concentrated and then poured into ice-cold water. The solid thus separated was filtered, washed with water and crystallized from glacial acetic acid to obtain the desired Compound. Dark yellow needles; yield: 52.2\%, $245-47^{\circ} \mathrm{C}$. The compounds $\mathbf{5}$ a-j were prepared similarly by treating with corresponding aldehydes.

(Z)-N-(5-(Benzylidene)-4-oxo-2-phenylthiazolidin-3-yl)4-(4-methylbenzamido)benzamide (5a): Dark yellow needles, yield: $52.21 \%$, mp $245-247{ }^{\circ} \mathrm{C}$, IR ( $\mathrm{KBr}, v_{\max }$, $\left.\mathrm{cm}^{-1}\right) 3341.98(\mathrm{~N}-\mathrm{H}), 3046.27(\mathrm{Ar}-\mathrm{H}), 2956.81\left(\mathrm{CH}_{3}\right)$, $1685.43(\mathrm{C}=\mathrm{O}$ in ring), $1630.12(\mathrm{C}=\mathrm{O}$ of amide), 703.29 (C$\mathrm{S}) ;{ }^{1} \mathrm{H} \mathrm{NMR}\left(400 \mathrm{MHz}, \mathrm{DMSO}-d_{6}\right) \delta 3.25\left(\mathrm{~s}, 3 \mathrm{H}, \mathrm{CH}_{3}\right)$, 7.06-7.75 (m, 18H, Ar-H), 8.08 (s, 1H, -CONH), 7.91 (s, $1 \mathrm{H}, \mathrm{CH}=\mathrm{C}) ;{ }^{13} \mathrm{C}$ NMR $\left(100 \mathrm{MHz}, \mathrm{DMSO}-d_{6}\right) \delta 25.71$ $\left(\mathrm{CH}_{3}\right), 60.28(-\mathrm{CH}), 48.51\left(\mathrm{C}_{5}\right.$ in thiazolidinone ring), 54.9 $(=\mathrm{CH}), 117.3,121.0,125.2,130.8,140.9,141.5$ (aromatics), 163.18 (amide- $\mathrm{C}=\mathrm{O}$ ), 159.2 (ring-C=O); MS: $m / z 520.72$ $[M+1]$.

(Z)-N-(5-(2-Chlorobenzylidene)-4-oxo-2-phenylthiazolidin-3-yl)-4-(4-methylbenzamido) benzamide (5b): Dark yellow needles; yield: $62.3 \%$; mp $250-254{ }^{\circ} \mathrm{C}$; IR ( $\mathrm{KBr}$, $\left.v_{\max }, \mathrm{cm}^{-1}\right) ; 3329.11(\mathrm{~N}-\mathrm{H}), 3053.21(\mathrm{C}-\mathrm{H}$ in ring), 3009.23 (Ar-H), $2956.53\left(\mathrm{CH}_{3}\right), 16835.04(\mathrm{C}=\mathrm{O}$ in ring), 1639.21 $(\mathrm{C}=\mathrm{O}), 1398.56(\mathrm{C}-\mathrm{N}), 1087.6(\mathrm{C}-\mathrm{Cl}) ;{ }^{1} \mathrm{H} \mathrm{NMR}(400 \mathrm{MHz}$, DMSO- $\left.d_{6}\right) \delta 3.19(\mathrm{~s}, 3 \mathrm{H} \mathrm{CH}), 7.06-7.8(\mathrm{~m}, 17 \mathrm{H}, \mathrm{Ar}-\mathrm{H})$; $7.98(\mathrm{~s}, 2 \mathrm{H}, \mathrm{CONH}), 7.91(\mathrm{~s}, 1 \mathrm{H}, \mathrm{C}=\mathrm{CH}) ;{ }^{13} \mathrm{C} \mathrm{NMR}(100$ $\left.\mathrm{MHz}, \mathrm{DMSO}-d_{6}\right) \delta 29.15\left(\mathrm{CH}_{3}\right), 62.51(-\mathrm{CH}), 50.03\left(\mathrm{C}_{5}\right.$ in thiazolidinone ring), $54.9(=\mathrm{CH}), 117.3,121.8,125.2,130.8$, 135.1, 140.2, 141.5 (aromatics), $162.21(\mathrm{C}=\mathrm{O}), 160.92(\mathrm{C}=\mathrm{O}$ in ring); MS: $m / z 555.91[M+2]$.

(Z)- $\mathrm{N}$-(5-(4-Methoxybenzylidene)-4-oxo-2-phenylthiazolidin-3-yl)-4-(4-methylbenzamido) benzamide (5c): Dark yellow needles; yield: 58.4\%; mp 241-244 ${ }^{\circ} \mathrm{C}$; IR ( $\mathrm{KBr}$, $\left.v_{\max }, \mathrm{cm}^{-1}\right) ; 3341.02(\mathrm{~N}-\mathrm{H}), 3009.23(\mathrm{Ar}-\mathrm{H}), 2943.56\left(\mathrm{CH}_{3}\right)$, $1681.28(\mathrm{C}=\mathrm{O}$ in ring $), 1639.21(\mathrm{C}=\mathrm{O}), 1398.56(\mathrm{C}-\mathrm{N})$, 1260.34 (C-O-C); ${ }^{1} \mathrm{H}$ NMR (400 MHz, DMSO- $d_{6}$ ) $\delta 3.25$ (s, $\left.3 \mathrm{H}, \mathrm{CH}_{3}\right), 3.91\left(\mathrm{~s}, 3 \mathrm{H}, \mathrm{OCH}_{3}\right), 7.10-7.78(\mathrm{~m}, 17 \mathrm{H}, \mathrm{Ar}-\mathrm{H})$, $7.85(\mathrm{~s}, 1 \mathrm{H}, \mathrm{CH}=\mathrm{C}), 7.98(\mathrm{~s}, 2 \mathrm{H}, \mathrm{CONH}) ;{ }^{13} \mathrm{C} \mathrm{NMR}(100$ $\left.\mathrm{MHz}, \mathrm{DMSO}-d_{6}\right) \delta 30.06\left(\mathrm{CH}_{3}\right), 62.51(-\mathrm{CH}), 52.19\left(\mathrm{C}_{5}\right.$ in thiazolidinone ring), $56.02(=\mathrm{CH}), 116.3,123,129.73$, 131.4, 140.2, 158.2 (aromatics), $163.18(\mathrm{C}=\mathrm{O}), 159.08$ $(\mathrm{C}=\mathrm{O}$ in ring); $\mathrm{MS}: m / z 550.41[M+1]$.

(Z)-4-Methyl- $N$-(4-((5-(2-nitrobenzylidene)-4-oxo-2-phenylthiazolidin-3-yl)carbamoyl)phenyl)benzamide (5d): Dark yellow needles; yield: $61.2 \%$; mp $251-254{ }^{\circ} \mathrm{C}$; IR $\left(\mathrm{KBr}, v_{\max }, \mathrm{cm}^{-1}\right) ; 3341.02(\mathrm{~N}-\mathrm{H}), 3009.23(\mathrm{C}-\mathrm{H}$ in $\mathrm{Ar})$, $2956.15\left(\mathrm{CH}_{3}\right), 1689.43(\mathrm{C}=\mathrm{O}$ in ring $), 1641.19(\mathrm{C}=\mathrm{O})$, $1525.61(\mathrm{~N}-\mathrm{O}), 1399.61(\mathrm{C}-\mathrm{N}), 701.22(\mathrm{C}-\mathrm{S}) ;{ }^{1} \mathrm{H} \mathrm{NMR}(400$ $\left.\mathrm{MHz}, \mathrm{DMSO}-d_{6}\right) \delta 8.11(\mathrm{~s}, 2 \mathrm{H}, \mathrm{O}=\mathrm{C}-\mathrm{N}-\mathrm{H}), 7.93(\mathrm{~s}, 1 \mathrm{H}$, $=\mathrm{CH}), 7.78\left(\mathrm{~d}, J=8.3 \mathrm{~Hz}, 2 \mathrm{H}, \mathrm{Ar}-\mathrm{H}\right.$ near $\left.\mathrm{NO}_{2}\right), 7.5(\mathrm{~d}, J=$ 8.6 Hz, 4H, Ar-H), 6.91-7.34 (m, 11H, Ar-H), 5.19 (s, 1H, $\mathrm{N}-\mathrm{CH}-\mathrm{Ar}), 2.78\left(\mathrm{~s}, 3 \mathrm{H}, \mathrm{CH}_{3}\right) ;{ }^{13} \mathrm{C}$ NMR (100 MHz, DMSO$\left.d_{6}\right) \delta 40.09\left(\mathrm{CH}_{3}\right), 64.28(-\mathrm{CH}), 52.19\left(\mathrm{C}_{5}\right.$ in thiazolidinone ring), $58.18(=\mathrm{CH}), 129.7,134.1,138.7,147.12$ (aromatics), $158.6(\mathrm{C}=\mathrm{O}$ in ring), $161.3(\mathrm{C}=\mathrm{O})$; $\mathrm{MS}: m / z 565.48[M+1]$.

(Z)-4-Methyl- $N$-(4-((4-0xo-2-phenyl-5-(3,4,5-trimethoxybenzylidene)thiazolidin-3-yl)carbamoyl)phenyl)benzamide (5e): Dark yellow needles; yield: 59.8\%; mp 221-223 ${ }^{\circ} \mathrm{C}$; IR $\left(\mathrm{KBr}, v_{\max }, \mathrm{cm}^{-1}\right)$; $3341.07(\mathrm{~N}-\mathrm{H}), 3006.13$ (Ar), 2949.31 $\left(\mathrm{CH}_{3}\right), 1681.68(\mathrm{C}=\mathrm{O}$ in ring $), 1640.21(\mathrm{C}=\mathrm{O}), 1272.49(\mathrm{C}-$ O-C), $1400.11(\mathrm{C}-\mathrm{N}) ;{ }^{1} \mathrm{H}$ NMR (400 MHz, DMSO-d $d_{6} \delta$ $8.13(\mathrm{~s}, 2 \mathrm{H}, \mathrm{O}=\mathrm{C}-\mathrm{N}-\mathrm{H}), 7.88(\mathrm{~s}, 1 \mathrm{H},=\mathrm{CH}), 7.09-7.53(\mathrm{~m}$, 13H, Ar-H), 6.89 (d, $J=9.1 \mathrm{~Hz}, 2 \mathrm{H}, \mathrm{Ar}-\mathrm{H}$ near $\left.\mathrm{OCH}_{3}\right), 5.27$ (s, 1H, N-CH-Ar), 3.46 (s, 6H, $\left.\mathrm{OCH}_{3}\right), 3.19$ (s, 3H, $\mathrm{OCH}_{3}$ ), $2.42\left(\mathrm{~s}, 3 \mathrm{H}, \mathrm{CH}_{3}\right) ;{ }^{13} \mathrm{C} \mathrm{NMR}\left(100 \mathrm{MHz}, \mathrm{DMSO}-d_{6}\right) \delta 40.09$ $\left(\mathrm{CH}_{3}\right), 52.19\left(\mathrm{C}_{5}\right.$ in thiazolidinone ring $), 58.18(=\mathrm{CH}), 59.9$ $\left(\mathrm{OCH}_{3}\right), 64.28(-\mathrm{CH}), 107.6,119.2,129.7,134.1,138.7$, 147.12 (aromatics), $158.6(\mathrm{C}=\mathrm{O}$ in $\operatorname{ring}), 161.3(\mathrm{C}=\mathrm{O})$; $\mathrm{MS}$ : $\mathrm{m} / \mathrm{z} 610.24[M+1]$.

(Z)-N-(5-(4-(Dimethylamino)benzylidene)-4-oxo-2-phenylthiazolidin-3-yl)-4-(4-methylbenzamido) benzamide (5f): Dark yellow needles; yield: 63.4\%; mp 249-251 ${ }^{\circ} \mathrm{C}$; IR $\left(\mathrm{KBr}, v_{\max }, \mathrm{cm}^{-1}\right) ; 3341.16(\mathrm{~N}-\mathrm{H}), 3055.01$ (C-H in ring), 3009.27 (Ar), $2956.67\left(\mathrm{CH}_{3}\right), 2932.72\left(\mathrm{CH}_{3}\right), 1691.01(\mathrm{C}=\mathrm{O}$ in ring), $1640.03(\mathrm{C}=\mathrm{O}), 1338.27(\mathrm{C}-\mathrm{N}) ;{ }^{1} \mathrm{H}$ NMR (400 MHz, DMSO-d $\left.d_{6}\right) \delta 2.78\left(\mathrm{~s}, 6 \mathrm{H}, \mathrm{CH}_{3}\right), 2.91\left(\mathrm{~s}, 3 \mathrm{H}, \mathrm{CH}_{3}\right)$, 7.08-7.75 (m, 17H, Ar-H), 7.86 (s, 1H, CH=C), 7.98 (s, 2H, $\mathrm{CONH}) ;{ }^{13} \mathrm{C}$ NMR $\left(100 \mathrm{MHz}, \mathrm{DMSO}-d_{6}\right) \delta 40.09\left(\mathrm{CH}_{3}\right)$, 
$64.28(-\mathrm{CH}), 52.19\left(\mathrm{C}_{5}\right.$ in thiazolidinone ring), $58.18(=\mathrm{CH})$, 114.8, 121.6, 129.8, 143.1, 150.02 (aromatics), $159.4(\mathrm{C}=\mathrm{O}$ in ring), $164.1(\mathrm{C}=\mathrm{O})$; MS: $m / z 563.39[M+1]$.

(Z)- $N$-(5-(4-Chlorobenzylidene)-4-oxo-2-phenylthiazolidin-3-yl)-4-(4-methylbenzamido) benzamide (5g): Dark yellow needles; yield: $60.8 \%$; mp $251-253{ }^{\circ} \mathrm{C}$; IR $(\mathrm{KBr}$, $\left.v_{\max }, \mathrm{cm}^{-1}\right) 3321.23(\mathrm{~N}-\mathrm{H}), 3057.29(\mathrm{C}-\mathrm{H}$ in ring), 3010.75 (Ar-H), $2951.93\left(\mathrm{CH}_{3}\right), 1690.11(\mathrm{C}=\mathrm{O}$ in ring), 1636.84 $(\mathrm{C}=\mathrm{O}), 1392.12(\mathrm{C}-\mathrm{N}), 1093.18(\mathrm{C}-\mathrm{Cl}), 701.88(\mathrm{C}-\mathrm{S}) ;{ }^{1} \mathrm{H}$ NMR (400 MHz, DMSO-d $\left.d_{6}\right) \delta 3.19\left(\mathrm{~s}, 3 \mathrm{H}, \mathrm{CH}_{3}\right), 7.06-7.8$ $(\mathrm{m}, 17 \mathrm{H}, \mathrm{Ar}-\mathrm{H}) ; 8.14$ (s, 2H, CONH), $7.94(\mathrm{~s}, 1 \mathrm{H}, \mathrm{C}=\mathrm{CH})$; ${ }^{13} \mathrm{C}$ NMR $\left(100 \mathrm{MHz}, \mathrm{DMSO}-d_{6}\right) \delta 29.15\left(\mathrm{CH}_{3}\right), 62.51$ $(-\mathrm{CH}), 50.03\left(\mathrm{C}_{5}\right.$ in thiazolidinone ring), $54.9(=\mathrm{CH}), 117.3$, 121.8, 125.2, 130.8, 135.1, 140.2, 141.5 (aromatics), 162.21 $(\mathrm{C}=\mathrm{O}), 160.92(\mathrm{C}=\mathrm{O}$ in ring $)$, MS: $m / z 555.93[M+2]$.

(Z)-4-Methyl- $N$-(4-((5-(4-methylbenzylidene)-4-oxo-2phenylthiazolidin-3-yl)carbamoyl)phenyl)benzamide (5h): Dark yellow needles; yield: 59.2\%; mp 249-250 ${ }^{\circ} \mathrm{C}$; IR $\left(\mathrm{KBr}, v_{\max }, \mathrm{cm}^{-1}\right) ; 3297.55(\mathrm{~N}-\mathrm{H}), 3052.11$ (C-H in ring), 3027.18 (Ar), $2955.12\left(\mathrm{CH}_{3}\right), 2949.16\left(\mathrm{CH}_{3}\right), 1683.09(\mathrm{C}=\mathrm{O}$ in ring), $1643.12(\mathrm{C}=\mathrm{O}), 1399.29(\mathrm{C}-\mathrm{N}), 701.82(\mathrm{C}-\mathrm{S}) ;{ }^{1} \mathrm{H}$ NMR (400 MHz, DMSO-d $) \delta 2.92\left(\mathrm{~s}, 6 \mathrm{H}, \mathrm{CH}_{3}\right), 7.08-7.8$ (m, 17H, Ar-H), 7.82 (s, 1H, CH=C), 7.94 (s, 2H, CONH); ${ }^{13} \mathrm{C}$ NMR (100 MHz, DMSO- $\left.d_{6}\right) \delta 40.09\left(\mathrm{CH}_{3}\right), 64.28(-\mathrm{CH})$, $52.19\left(\mathrm{C}_{5}\right.$ in thiazolidinone ring $), 58.18(=\mathrm{CH}), 114.8,121.6$, 129.8, 143.1, 150.02 (aromatics); MS: $m / z 534.41[M+1]$.

(Z)-4-Methyl- $N$-(4-((5-(4-nitrobenzylidene)-4-oxo-2-phenylthiazolidin-3-yl)carbamoyl)phenyl)benzamide (5i): Dark yellow needles; yield: 59.9\%; mp 249-251 ${ }^{\circ} \mathrm{C}$; IR $\left(\mathrm{KBr}, v_{\max }, \mathrm{cm}^{-1}\right) ; 3341.02(\mathrm{~N}-\mathrm{H}), 3009.91(\mathrm{C}-\mathrm{H}$ in $\mathrm{Ar})$, $2954.78\left(\mathrm{CH}_{3}\right), 1679.28(\mathrm{C}=\mathrm{O}$ in ring $), 1641.19(\mathrm{C}=\mathrm{O})$, $1525.61(\mathrm{~N}-\mathrm{O}), 1399.61(\mathrm{C}-\mathrm{N}), 701.22(\mathrm{C}-\mathrm{S}) ;{ }^{1} \mathrm{H}$ NMR $(400$ $\left.\mathrm{MHz}, \mathrm{DMSO}-d_{6}\right) \delta 8.11(\mathrm{~s}, 2 \mathrm{H}, \mathrm{O}=\mathrm{C}-\mathrm{N}-\mathrm{H}), 7.93(\mathrm{~s}, 1 \mathrm{H}$, $=\mathrm{CH}), 7.71\left(\mathrm{~d}, J=8.3 \mathrm{~Hz}, 2 \mathrm{H}, \mathrm{Ar}-\mathrm{H}\right.$ near $\left.\mathrm{NO}_{2}\right), 7.5(\mathrm{~d}, J=$ $8.3 \mathrm{~Hz}, 4 \mathrm{H}, \mathrm{Ar}-\mathrm{H}), 6.91-7.35(\mathrm{~m}, 11 \mathrm{H}, \mathrm{Ar}-\mathrm{H}), 5.19$ (s, 1H, $\mathrm{N}-\mathrm{CH}-\mathrm{Ar}), 2.73$ (s, 3H, $\left.\mathrm{CH}_{3}\right) ;{ }^{13} \mathrm{C}$ NMR (100 MHz, DMSO$\left.d_{6}\right) \delta 40.09\left(\mathrm{CH}_{3}\right), 64.28(-\mathrm{CH}), 52.19\left(\mathrm{C}_{5}\right.$ in thiazolidinone ring), $58.18(=\mathrm{CH}), 129.1,133.5,138.7,147.12$ (aromatics), $158.6(\mathrm{C}=\mathrm{O}$ in ring), $162.1(\mathrm{C}=\mathrm{O})$. $\mathrm{MS}: m / z 565.48[M+1]$.

(Z)- $N$-(5-(4-Bromobenzylidene)-4-oxo-2-phenylthiazolidin-3-yl)-4-(4-methylbenzamido) benzamide (5j): Dark yellow needles; yield: $57.2 \%$; mp $238-241{ }^{\circ} \mathrm{C}$; IR ( $\mathrm{KBr}$, $\left.v_{\max }, \mathrm{cm}^{-1}\right) ; 3341.02(\mathrm{~N}-\mathrm{H}), 3009.91(\mathrm{C}-\mathrm{H}$ in $\mathrm{Ar}), 2952.54$ $\left(\mathrm{CH}_{3}\right), 1685.27(\mathrm{C}=\mathrm{O}$ in ring $), 1641.19(\mathrm{C}=\mathrm{O}), 1399.61(\mathrm{C}-$ $\mathrm{N}), 701.22(\mathrm{C}-\mathrm{S}) .{ }^{1} \mathrm{H}$ NMR (400 MHz, DMSO-d 6 ) $\delta 8.02(\mathrm{~s}$, $2 \mathrm{H}, \mathrm{O}=\mathrm{C}-\mathrm{N}-\mathrm{H}), 7.93(\mathrm{~s}, 1 \mathrm{H},=\mathrm{CH}), 7.5(\mathrm{~d}, J=8.6 \mathrm{~Hz}, 4 \mathrm{H}$, Ar-H), 6.91-7.59 (m, 13H, Ar-H), 5.13 (s, 1H, N-CH-Ar), $2.85\left(\mathrm{~s}, 3 \mathrm{H}, \mathrm{CH}_{3}\right) ;{ }^{13} \mathrm{C}$ NMR (100 MHz, DMSO- $\left.d_{6}\right) \delta 40.09$ $\left(\mathrm{CH}_{3}\right), 64.28(-\mathrm{CH}), 52.19\left(\mathrm{C}_{5}\right.$ in thiazolidinone ring), 58.18 $(=\mathrm{CH}), 128.2$, 129.1, 133.5, 138.7 (aromatics), $157.2(\mathrm{C}=\mathrm{O}$ in ring), $163.4(\mathrm{C}=\mathrm{O})$. MS: $m / z 600.12[M+1]$.

Antimicrobial Studies Procedure. The newly prepared compounds 5a-j were screened for their anti microbial activity against Bacillus subtilis, Bacillus thuringiensis, Escherichia coli and Pseudomonas aeruginosa bacterial strains and Candida albicans, Botrytis fabae and Fusarium oxysporam fungal strains were used This activity was deter- mined by agar diffusion method. Compounds were dissolved in DMSO at concentration $1 \mathrm{mg} \mathrm{mL}^{-1}$.

The antibacterial and antifungal activities of each compound were compared with Streptomycin and Treflucan as the standard drugs.

The results were recorded for each tested compound as the average diameter of inhibition zones (IZ) of bacterial or fungal growth around the disks in $\mathrm{mm}$. The minimum inhibitory concentration (MIC) measurement was showed significant growth inhibition zones $(>10 \mathrm{~mm})$ using twofold serial dilution method.

Anti Tubercular Studies Procedure. All the synthesized compounds of series $\mathbf{5 a - j}$ were evaluated for their anti tubercular activity. Drug susceptibility and determination of MIC of the test compounds against M. tuberculosis H37Rv were performed by agar micro dilution method, where two fold dilutions of each test compound were added into 7H10 agars supplemented with OADC and organism. A culture of used microorganism M. tuberculosis $\mathrm{H} 37 \mathrm{Rv}$ growing on L-J medium was harvested in $0.85 \%$ saline with $0.05 \%$ Tween80. A suspension of compounds was prepared in DMSO. This suspension was added to (in tubes) $7 \mathrm{H} 10$ middle brook's medium (containing $1.7 \mathrm{~mL}$ medium and $0.2 \mathrm{~mL}$ OADC supplement) at different concentrations of compound keeping the volume constant, that is, $0.1 \mathrm{~mL}$ medium was allowed to cool keeping the tubes in slanting position. These tubes were then incubated at $37^{\circ} \mathrm{C}$ for $24 \mathrm{~h}$ followed by streaking of $M$. tuberculosis $\mathrm{H} 37 \mathrm{Rv}\left(5 \times 10^{4}\right.$ bacilli per tube). These tubes were then incubated at $37^{\circ} \mathrm{C}$. Growth of bacilli was seen after 30 days of incubation. Tubes having the compounds were controlled with control tubes where medium alone was incubated with H37Rv. The concentration at which complete inhibition of colonies occurred was taken as active concentration of test compound. Isoniazid was used as standard drug. The MIC levels of some active compounds 5a-j against these organisms were given in Table 2 .

\section{Results and Discussion}

Chemistry. In the present work, a series of new moieties was synthesized. Scheme 1 describes the path used for the preparation of target compounds. The key intermediate, 4 required for the synthesis of title compounds was prepared according to the procedure outlined in the Scheme 1. For the synthesis of required reaction sequence including benzoylation of benzocaine at the first step, which involves the protection of the amino function gave 4-(4-Methyl-benzoylamino)-benzoic acid ethyl ester (1). Hydrazinolysis of compound 1 then gave $N$-(4-hydrazinocarbonyl-phenyl)-4methyl-benzamide (2). This was later reacted with benzaldehyde in the presence of glacial. AcOH in methanol at reflux temperature gave 4-(Benzylidene-hydrazino[ $N$-4methyl-benzamide]Carbonyl)-phenyl (3). Compound 3 was then reacted with thioglycolic acid in the presence of anhydrous $\mathrm{ZnCl}_{2}$ under conventional heating conditions (Scheme 1) to give compound $\mathbf{4}$. Compound $\mathbf{4}$ was reacted with various aromatic aldehydes in the presence of anhydr- 
<smiles>CCOC(=O)c1ccc(NC(=O)c2ccc(C)cc2)cc1</smiles>

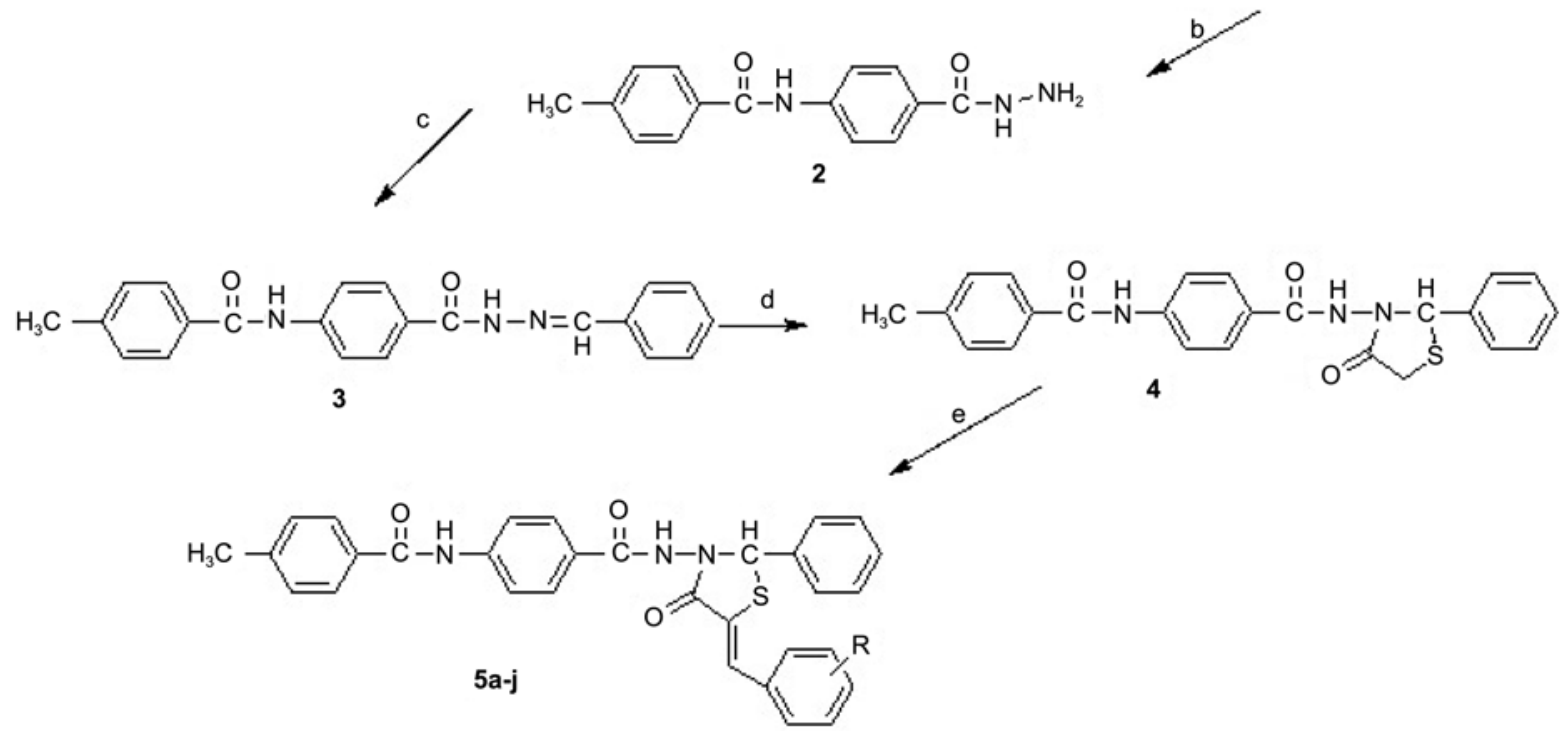

$\mathbf{5 a} \mathrm{R}=\mathrm{H}, \mathbf{5 b} \mathrm{R}=2-\mathrm{Cl}, \mathbf{5} \mathbf{c} \mathrm{R}=4-\mathrm{OH}, \mathbf{5 d} \mathrm{R}=2-\mathrm{NO}_{2}, \mathbf{5 e} \mathrm{R}=3,4,5-\left(\mathrm{OCH}_{3}\right)_{3}, \mathbf{5 f} \mathrm{R}=4-\mathrm{N}\left(\mathrm{CH}_{3}\right)_{2}, \mathbf{5} \mathbf{g} \mathrm{R}=4-\mathrm{Cl}, \mathbf{5 h} \mathrm{R}=4-\mathrm{CH}_{3}, \mathbf{5 i} \mathrm{R}=4-\mathrm{NO}_{2}, \mathbf{5 j} \mathrm{R}=4-\mathrm{Br}$

Scheme 1. Synthesis of compounds 5a-j.

Reaction conditions: a) Dry ether, $0^{\circ} \mathrm{C}$, b) $\mathrm{NH}_{2} \mathrm{NH}_{2} \cdot \mathrm{H}_{2} \mathrm{O}$, ethanol, c) Benzaldehyde, glacial $\left.\mathrm{AcOH}, \mathrm{d}\right) \mathrm{SHCH} \mathrm{COOH}_{2} \mathrm{DMF}$, anhydrous $\mathrm{ZnCl}_{2}$, e) aromatic aldehydes, anhydrous $\mathrm{CH}_{3} \mathrm{COONa}$, glacial $\mathrm{AcOH}$.

ous $\mathrm{NaOAc}$ in glacial acetic acid at reflux temperature to give chalcone derivatives of thiazolidinones 5 . The structures of the synthesized compounds were confirmed by IR, ${ }^{1} \mathrm{H}$ $\mathrm{NMR},{ }^{13} \mathrm{C}$ NMR and mass spectral analysis.

In the IR spectra of compounds 5a-j disappearance of active methylene group absorption band at $2914.73 \mathrm{~cm}^{-1}$, which was present in compound $\mathbf{4}$, confirmed the chalcone derivatives of Thiazolidinone system.

In the ${ }^{1} \mathrm{H}$ NMR spectra of compounds $\mathbf{5 a}-\mathbf{j}$, recorded in DMSO- $d_{6}$, The disappearance of active methylene group signal at $4.03 \mathrm{ppm}$ as a singlet, which was present in compound 4, as well as the appearance of various signals due to $=\mathrm{CH}$ protons appeared at $7.9 \mathrm{ppm}$ as a singlet and the $\mathrm{N}-\mathrm{CH}-\mathrm{S}$ proton of thiazolidinone ring at $5.51-5.65 \mathrm{ppm}$ as a singlet demonstrate that the condensation step had occurred. All the other aromatic protons of 5a-j were observed at the expected regions. In the ${ }^{13} \mathrm{C}$ NMR spectrum of compounds $\mathbf{5 a - j}$, recovered in DMSO- $d_{6}$, the prominent signals corresponding to the carbons of chalcone derivatives of thiazolidinone moiety observed nearly at 25.71, 48.51, 54.9, 60.28,

Table 1. Anti bacterial and anti fungal data of compounds 5a-j

\begin{tabular}{|c|c|c|c|c|c|c|c|}
\hline \multirow{2}{*}{ Compound No. } & \multicolumn{4}{|c|}{ Bacteria $^{b}$} & \multicolumn{3}{|c|}{ Fungi $^{c}$} \\
\hline & B. subtilis & B. thuringiensis & E. coli & P. aeruginosa & B. fabae & F. oxysporam & C. albicans \\
\hline $5 a$ & 12.5 & 12.5 & 12.5 & 25 & 25 & 25 & 12.5 \\
\hline $5 \mathbf{b}$ & 3.125 & 3.125 & 6.25 & 6.25 & 12.5 & 12.5 & 6.25 \\
\hline $5 c$ & 25 & 25 & 25 & 50 & 6.25 & 3.125 & 3.125 \\
\hline $5 d$ & 3.125 & 3.125 & 3.125 & 3.125 & 25 & 25 & 25 \\
\hline $5 e$ & 25 & $\mathrm{NA}^{a}$ & $\mathrm{NA}^{a}$ & 25 & 3.125 & 3.125 & 3.125 \\
\hline $5 f$ & 6.25 & 12.5 & 12.5 & $\mathrm{NA}^{a}$ & 12.5 & 12.5 & 25 \\
\hline $5 \mathrm{~g}$ & 3.125 & 3.125 & 3.125 & 6.25 & 25 & $\mathrm{NA}^{a}$ & 12.5 \\
\hline $5 \mathrm{~h}$ & 25 & 25 & $\mathrm{NA}^{a}$ & $\mathrm{NA}^{a}$ & 3.125 & 6.25 & 3.125 \\
\hline $5 i$ & 3.125 & 3.125 & 6.25 & 6.25 & 12.5 & 6.25 & 6.25 \\
\hline $5 j$ & 6.25 & 6.25 & 6.25 & 6.25 & $\mathrm{NA}^{a}$ & 25 & 12.5 \\
\hline Strepto mycin & 3.125 & 6.25 & 6.25 & 6.25 & $\mathrm{NA}^{a}$ & $\mathrm{NA}^{a}$ & $\mathrm{NA}^{a}$ \\
\hline Treflucan & $\mathrm{NA}^{a}$ & $\mathrm{NA}^{a}$ & $\mathrm{NA}^{a}$ & $\mathrm{NA}^{a}$ & 3.125 & 3.125 & 3.125 \\
\hline
\end{tabular}

${ }^{a}$ Not Active. ${ }^{b} B$. subtilis (MTCC No: 1133), B. thuringiensis (MTCC No: 4714), E. coli (MTCC No: 443), and P. aeruginosa (MTCC No: 2297 ). ${ }^{c} B$ fabae (ATCC No: 14862), F. oxysporam (MTCC No: 7392) and C. albicans (MTCC No: 183) 
$130.8,167.18$ and $171.72 \mathrm{ppm}$ are proof of further evidence of their structures.

\section{Pharmacology.}

Anti Microbial Studies: The results depicted in Table 1 revealed that most of tested compounds displayed variable inhibitory effects on the growth of tested gram positive, gram negative bacterial strains and fungal strains.

The anti bacterial screening data showed moderate activity of the test compounds. Among the screened 5b, 5d, $\mathbf{5 g}, 5 \mathbf{i}$ and $5 \mathbf{j}$ in which chalcone derivatives of thiazolidinones bearing $o$-chlorophenyl, $o$-nitrophenyl, $p$-chlorophenyl, $p$-nitrophenyl and $p$-bromophenyl nucleus respectively show high activity due to the presence of benzoyl moiety against all the microorganisms employed. In this view compound $\mathbf{5 b}$ and $\mathbf{5 i}$ were equipotent to streptomycin against B. subtilis (MIC, $3.125 \mu \mathrm{g} / \mathrm{mL}$ ), E. coli (MIC, 6.25 $\mu \mathrm{g} / \mathrm{mL}$ ) and $P$. aeruginosa (MIC, $6.25 \mu \mathrm{g} / \mathrm{mL}$ ), while its activity was more potent to streptomycin against $B$. thuringiensis (MIC, $3.125 \mu \mathrm{g} / \mathrm{mL}$ ). In the same way compound $\mathbf{5 d}$ was more potent than streptomycin against all the strains employed except B. subtilis. Besides this compound $\mathbf{5 g}$ was equipotent to streptomycin against $B$. subtilis (MIC, 3.125 $\mu \mathrm{g} / \mathrm{mL}$ ) and $P$. aeruginosa (MIC, $6.25 \mu \mathrm{g} / \mathrm{mL}$ ), while its activity was $50 \%$ more potent to streptomycin against all other bacterial strains employed (MIC, $3.125 \mu \mathrm{g} / \mathrm{mL}$ ) and the compound $\mathbf{5 j}$ was equipotent to streptomycin against all the strains employed except B. subtilis (MIC, $6.25 \mu \mathrm{g} / \mathrm{mL}$ ). Finally the activity of these compounds showed moderate to good antibacterial activity.

Regarding the activity of thiazolidinones and their 5arylidenes derivatives incorporating benzoyl moiety against antifungal strains the results revealed that compounds $\mathbf{5 c}, \mathbf{5 e}$, $\mathbf{5 h}$ and $\mathbf{5 i}$ revealed strong growth inhibitory against the tested fungi. In this view compound $\mathbf{5 c}$ was equipotent to treflucan against $F$. oxysporam and $C$. albicans (MIC, 3.125 $\mu \mathrm{g} / \mathrm{mL}$ ), while its activity was $50 \%$ less potent to treflucan against $B$. fabae (MIC, $6.25 \mu \mathrm{g} / \mathrm{mL}$ ). And compound 5e was equipotent to treflucan against all the strains employed (MIC, $3.125 \mu \mathrm{g} / \mathrm{mL}$ ). On the other hand $5 \mathrm{~h}$ was equipotent to treflucan against B. fabae and C. albicans (MIC, 3.125 $\mu \mathrm{g} / \mathrm{mL}$ ), while its activity was $50 \%$ less potent to treflucan

Table 2. Anti tubercular activity data of compounds $\mathbf{5 a - j}$

\begin{tabular}{cc}
\hline Compound & MIC $(\mu \mathrm{g} / \mathrm{mL})$ \\
\hline $\mathbf{5 a}$ & $>25$ \\
$\mathbf{5 b}$ & $>3.125$ \\
$\mathbf{5 c}$ & $>25$ \\
$\mathbf{5 d}$ & 6.25 \\
$\mathbf{5 e}$ & $>25$ \\
$\mathbf{5 f}$ & $>12.5$ \\
$\mathbf{5 g}$ & $>6.25$ \\
$\mathbf{5 h}$ & $>12.5$ \\
$\mathbf{5 i}$ & $>3.125$ \\
$\mathbf{5 j}$ & 6.25 \\
Streptomycin & 4.0 \\
\hline
\end{tabular}

against $F$. oxysporam (MIC, $6.25 \mu \mathrm{g} / \mathrm{mL}$ ). Finally the activity of these compounds showed moderate to good antifungal activity.

Anti Tubercular Studies: The results depicted in Table 2 revealed that most of the tested compounds, displayed variable inhibitory effects on the growth of the tested $M$. tuberculosis H37Rv strains.

Generally compounds possessing electron-withdrawing groups showed good anti tubercular activity. Some derivatives (5b, 5d, 5g, 5i and 5j) containing electron-withdrawing groups $\left(-\mathrm{Cl},-\mathrm{NO}_{2},-\mathrm{Br}\right)$ have shown promising activity against $\mathrm{M}$. tuberculosis.

\section{Conclusion}

In conclusion, the obtained results clearly revealed that some of the newly synthesized compounds where the benzoyl moiety attached to arylidene-substituted thiazolidinones $(\mathbf{5 b}, \mathbf{5 d}, \mathbf{5 g}$ and $\mathbf{5 i})$ exhibited promising antimicrobial, anti tubercular activity.

Acknowledgments. The authors are grateful to The Head, Department of Chemistry, Dr. H. S. Gour University Sagar for providing necessary facilities to carry out this work. We sincerely express our gratitude to Head, Biotechnology laboratory, Department of Botany, Dr. H. S. Gour University Sagar for providing antimicrobial data. We are grateful to The Director, SAIF, Panjab University for providing spectral data and The Director Micro care laboratory Surat, for providing anti tubercular data.

\section{References}

1. Khan, S. A.; Yousuf, M. Eur. J. Med. Chem. 2009, 44, 2597.

2. Palekar, V. S.; Damle, A. J.; Shukla, S. R. Eur. J. Med. Chem. 2009, 44, 5112.

3. Kucukguzel, S. G.; Oruc, E. E.; Rollas, S.; Sahin, F.; Ozbek, A. Eur. J. Med. Chem. 2002, 37, 197.

4. Bonde, C. G.; Gaikwad, N. J. Bioorg. Med. Chem. 2004, 12, 2151.

5. Bhatt, J. J.; Shah, B. R.; Shah, H. P.; Trivedi, P. B.; Undavia, N. K.; Desai, N. C. Ind. J. Chem. 1994, 33B, 189.

6. Babaoglu, K.; Page, M. A.; Jones, V. C.; Mc Neil, M. R.; Dong, C.; Naismith, J. H.; Lee, R. E. Bioorg. Med. Chem. Lett. 2003, 13, 3227.

7. Ulusoy, N. Forsch, A. Drug Res. 2002, 52, 565.

8. Hogale, M.; Uthale, A. Proc. Ind. Acad. Sci. 1990, 102, 535.

9. Yadav, R.; Srivastava, S. D.; Srivastava, S. K. Ind. J. Chem. 2005, 44B, 1262.

10. Barreca, M. L.; Chimirri, A.; Luca, L. D.; Monforte, A. M.; Monforte, P.; Rao, A.; Jappala, M.; Balzarini, J.; Clercq, E. De.; Pannecouque, C.; Witvrouw, M. Bioorg. Med. Chem. Lett. 2001, $11,1793$.

11. Vigorita, M. G.; Ottana, R.; Monforte, F.; Maccari, R.; Trovato, A.; Monforte, M. T.; Taviano, M. F. Bioorg. Med. Chem. Lett. 2001, 11, 2791 .

12. Abdel-Wahab, B. F.; Awad, G. E. A.; Badria, F. A. Eur. J. Med Chem. 2011, 46, 1505.

13. Havrylyuk, D.; Mosula, L.; Zimenkovsky, B.; Vasylenko, O.; Gzella, A.; Lesyk, R. Eur. J. Med. Chem. 2010, 45, 5012.

14. Lakhan, R.; Singh, R. L. Proc. Indian Acad. Sci. (Chem. Sci.) 1991, 33, 103 . 